\title{
Optmization of the beta-beam baseline
}

\author{
M. Benedikt ${ }^{\mathrm{a}}$, A. Fabich ${ }^{\mathrm{a}}, \mathrm{S} . \mathrm{Hancock}^{\mathrm{a}}, \mathrm{M}$. Lindroos $^{\mathrm{a}}$ and The beta-beam task within EURISOL DS ${ }^{\mathrm{b} *}$ \\ aA-department, CERN, CH-1211 Geneva 23, Switzerland \\ bhttp://eurisol.org
}

The beta-beam concept for the production of intense electron (anti-)neutrino beams is now well established. A baseline design has recently been published for a beta-beam facility at CERN. It has the virtue to respect the known limitations of the PS and SPS synchrotrons at CERN but it falls short of delivering the requested annual rate of anti-neutrinos. We here report on a first analysis on how to increase the rate.

\section{Introduction}

A powerful method to understand the functional dependence of the many parameters influencing the figure of merit for a certain facility is available with modern analytical calculation software. The method requires that a symbolic analytical description is produced of the full accelerator chain and such a description has been done for the proposed beta-beam facility at CERN using Mathematica [5-7]. The results can be visualized as e.g. two-dimensional plots for the figure of merit as function of the different machine parameters. However, it is important to note that while such plots will help to identify the right parameter space for a requested machine design, they do not themselves guarantee that a realizable technical solution can be found. The work presented here has been done respecting the constraint of using the existing CERN accelerators, PS and SPS. In addition, the current decay ring design [3] is used even though it has been assumed that the Lorenz gamma of the stored ions can be changed without major changes to the lattice of the decay ring. For the stacking we have assumed that $15{ }^{6} \mathrm{He}$ bunches and $20{ }^{18} \mathrm{Ne}$ bunches can be merged in the decay ring without major losses. The situation is slightly better for ${ }^{18} \mathrm{Ne}$, which due to a more advantageous charge-to-mass ra-

*Project supported by EC under EURISOL DS Contract no. 515768 RIDS. The EC is not liable for any use that may be made of the information contained herein. tio, will see an almost three times larger longitudinal acceptance of the decay ring. Theoretically this would permit up to 45 merges for ${ }^{18} \mathrm{Ne}$, but known limits in low level RF beam control precision restricts it to a maximum of 20 merges. All parameters for the EURISOL DS baseline of May 2005 are documented in the appendixes of $[6,7]$.

\section{Annual rate}

The figure of merit for the beta-beam is the rate $(R)$ of (anti-)neutrinos at the end of one of the straight sections over a given period and it can be expressed as,

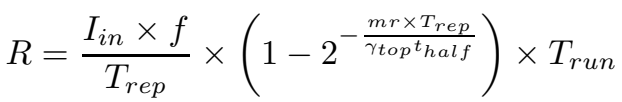

where the $m r$ is the number of merges that can be done in the decay ring without major losses from the merging process itself, $T_{r e p}$ is the repetition period for the fills in the decay ring, $\gamma_{t o p}$ the gamma factor of the decay ring, $t_{\text {hal } f}$ the half life at rest for the ions, $I_{\text {in }}$ the total number of ions injected into the decay ring for each fill, $f$ the fraction of the decay ring length for the straight section generating the neutrino beam and $T_{\text {run }}$ the length in seconds of the run. The first term gives the rate per chosen period for the ideal decay ring in which an endless number of merges can be accepted, the second term gives the limit set by the restriction on maximum number of merges that can be accepted due to longitudinal emittance limitations and the third term is by conven- 
tion chosen as the length of a "snow mass year" [4] which is used as an international standard to calculate running time of high energy physics experiments and it is $10^{7}$ seconds long.

\section{Gamma dependence of the CERN base- line}

The gamma dependence of the rate is mainly due to the gamma dependence of the acceleration time in the SPS, the lifetime of the ions in the decay ring and of the available longitudinal acceptance in the decay ring. The acceleration time in the SPS has a minimum length depending on hardware limitations and also increases in steps of 1.2 seconds due to the basic timing period of the CERN accelerator complex. For a given radiofrequency $(\mathrm{RF})$ voltage, the longitudinal acceptance will to first order scale as the square root of gamma. In figure 1 dependence of ${ }^{6} \mathrm{He}$ is shown. The maximum gamma that can be reached with the CERN SPS for ${ }^{6} \mathrm{He}$ is 150 and for ${ }^{18} \mathrm{Ne} 250$.

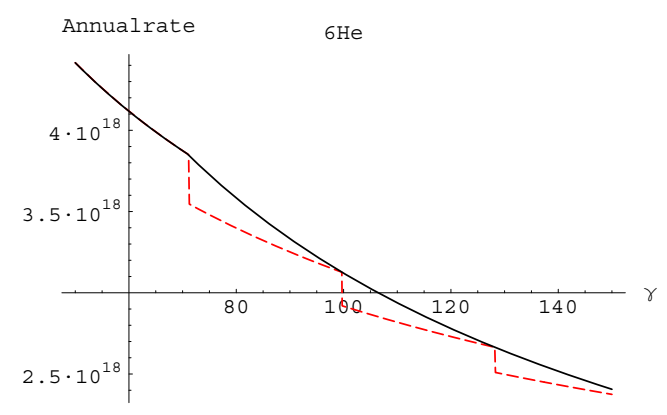

Figure 1. The annual rate of anti-neutrinos from ${ }^{6} \mathrm{He}$ as a function of gamma. The red dashed line shows the annual rate with the given source rate respecting a basic period of 1.2 seconds of the CERN accelerator complex. The solid line shows the same dependence but with a "smooth" choice of acceleration time for PS and SPS.

\subsection{Duty cycle dependence}

The available longitudinal acceptance for stacking in the decay ring can be increased by increasing the number of bunches in the decay ring. This will increase the duty factor which is the total length in time of all bunches in the decay ring divided by the revolution time. The present limits of 15 merges for ${ }^{6} \mathrm{He}$ and 20 merges for ${ }^{18} \mathrm{Ne}$ will truncate the stacking well before decay rate equals the stacking rate. In the formula for the annual rate (1) the second term is the result of this truncation. The duty cycle in the beta-beam baseline is $4.5 \times 10^{-3}$ for 20 bunches ${ }^{6} \mathrm{He}$ and $3.9 \times 10^{-3}$ for 20 bunches of ${ }^{18} \mathrm{Ne}$ at a gamma of 100 . Figure 2 shows the annual rate for ${ }^{18} \mathrm{Ne}$ as a function of number of bunches in the decay ring. $[6,7]$. The constraint on the duty-cycle is set by

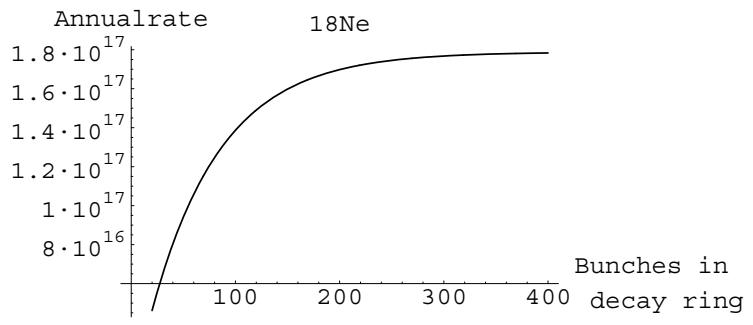

Figure 2. The annual rate of anti-neutrinos from the decay of ${ }^{18} \mathrm{Ne}$ as a function of number of bunches. The curve starts to the left with 8 bunches at a duty factor of $2 \times 10^{-3}$ and saturates when the decay rate equals the stacking rate.

the atmospheric background and it becomes less severe at higher energies of the neutrinos. A betabeam facility operating at a higher gamma than the nominal value of 100 could accept a larger duty factor. Consequently, to fully understand the evolution of the annual rate as a function of gamma the possibility to increase the duty factor 
must be fully explored.

\subsection{Accumulation}

In the beta-beam baseline the ions are produced for one second and thereafter accelerated during several seconds to a $\gamma$ of 100 . In theory, ions could be produced continuously as long as they can be stored while waiting for acceleration. The optimum storage time determined by the lifetime of the ion and the required acceleration time as can be seen in the curve in figure 3. In these calculations the accumulation is done before the PS in a storage ring fitted with a cooling system for an efficient storage through multiple injection cycles [9].

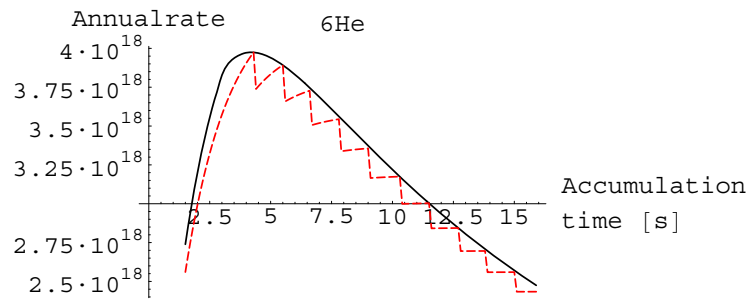

Figure 3. The annual rate of anti-neutrinos from ${ }^{6} \mathrm{He}$ as a function of the accumulation time in a storage ring before the PS. The red dashed line shows the annual rate respecting a basic period of 1.2 seconds of the CERN accelerator complex. The solid line shows the same dependence but with a "smooth" choice of acceleration time for PS and SPS. All other parameters have been taken from the baseline design

\section{Conclusions}

It has already been established that the increase of production rate of radioactive species translates linearly into an increase of the neutrino rate [10]. We have demonstrated that an increase of the duty cycle permits more particles to be stored in the decay ring and that accumulation can potentially be a powerful tool to make better use of the ions produced. The gamma dependence of the rate is often forgotten in physics reach calculations, but it should be noted that it is linked to longitudinal acceptance and duty factor and has to be calculated specifically for each choice of machine parameters.

\section{REFERENCES}

1. P.Zucchelli, A novel concept for a neutrino factory: the beta-beam, Phys. Let. B, 532 (2002) 166-172

2. B.Autin,M. Benedikt, M. Grieser, S. Hancock, H. Haseroth, A. Jansson, U. Kster, M. Lindroos, S. Russenschuck and F. Wenander, The acceleration and storage of radioactive ions for a neutrino factory, CERN/PS 2002078 (OP), Nufact Note 121, Proceedings of Nufact 02, London, UK, 2002, J. Phys. G: Nucl. Part. Phys. 29 (2003) 1785-1795

3. A.Chance and J. Payet, "Studies of the injection system in the decay ring of the betabeam neutrino source", Proceedings of 2005 Particle Accelerator Conference, Knoxville, Tennessee, http://www.jacow.org

4. http://snowmass2001.org/

5. (c)Wolfram Research, Inc., http://www.wolfram.com

6. M. Benedikt, S. Hancock, M. Lindroos, "Parameter and Intensity Values, Version 1, April 2005", EURISOL DS/TASK12/TN-0501, http://eurisol.org

7. M. Benedikt, A. Fabich, S. Hancock, M. Lindroos, "Parameter and Intensity Values, Version 2, July 2005", EURISOL DS/TASK12/TN-05-03, http://eurisol.org

8. "The EURISOL report", Edt. J. Cornell, GANIL, Caen, 2003, European commission contract No. HPRI-CT-1999-500001

9. T. Fritioff, M. Grieser, M. Lindroos, A. Trzcinska, Nucl. Phys. A 746(2004)384c-388c

10. M. Lindroos, "The technical challanges of a beta-beam", In these proceedings. 